\title{
Tratamiento endoscópico para el reflujo gastroesofágico
}

\author{
Ítalo Braghetto M. ${ }^{1}$
}

\section{Endoscopic treatment of gastroesophageal reflux}

During the last 2 decades, new endoscopic treatments have been developed for the treatment of gastroesophageal reflux disease (GERD) as alternatives to medical treatment or surgical fundoplication. These devices include application of radiofrequency treatment (Stretta), endoscopic plication (EndoCinch, Plicator, Esophyx, MUSE) and injection or implantation of biomaterials (Enteryx, Gatekeeper, Plexiglas, Duragel). Its objective is the relief of symptoms by creating an anatomical anti-reflux barrier. In this review, we consider articles indexed in Pubmed, Medline and Scielo in the last 10 years, reviewing a total of 55 papers, we analyse critically the reported results, although long-term data greater than 5 or 7 years are lacking. These procedures reduce the use of proton pump inhibitors by about $50 \%$. Subjective evaluations report improvement in quality of life and patient satisfaction. However, objective evaluation with endoscopy, manometry, radiology and pHmetry are scarce and if there are, they do not show significant changes. There is no convincing evidence to adopt these methods as definitive treatment. Therefore, laparoscopic fundoplication is the gold standard for the treatment of gastroesophageal reflux disease.

Keywords: endoscopy; gastroesophageal reflux; treatment.

\section{Resumen}

Durante las últimas 2 décadas se han desarrollado una serie de nuevos tratamientos endoscópicos para el tratamiento de la enfermedad por reflujo gastroesofágico (ERGE) como alternativas al tratamiento médico o funduplicatura quirúrgica. Estos dispositivos incluyen aplicación de tratamiento por radiofrecuencia (Stretta), plicatura endoscópica (EndoCinch, Plicator, Esophyx, MUSE) e inyección o implantación de biomateriales (Enteryx, Gatekeeper, Plexiglas, Duragel). Su objetivo es el alivio de los síntomas creando una barrera anatómica antirreflujo. En esta revisión del tema consideramos artículos indexados en Pubmed, Medline y Scielo en los últimos 10 años revisando un total de 55 trabajos. Evaluamos críticamente los resultados reportados, faltan datos a largo plazo superiores a 10 años. Estos procedimientos reducen el uso de inhibidores de la bomba de protones en cerca del 50\%. Evaluaciones subjetivas reportan mejoría de la calidad de vida y satisfacción del paciente. Sin embargo, la evaluación objetiva con endoscopia, manometría, radiología y pHmetría son escasos y si los hay, no muestran cambios significativos. No existe evidencia convincente para adoptar estos métodos como tratamiento definitivo y, por lo tanto, la fundoplicatura por vía laparoscópica sigue siendo el estándar de oro para el tratamiento de la enfermedad por reflujo gastroesofágico.

Palabras clave: endoscopía; reflujo gastroesofágico; tratamiento.

\section{Introducción}

Para la indicación terapéutica adecuada de un paciente con enfermedad por reflujo gastroesofágico, el estudio objetivo evaluando las características manométricas del esfínter gastroesofágico, la motilidad esofágica, existencia o no de hernia de hiato $(\mathrm{HH})$ y que tipo de hernia de acuerdo con los estudios de imágenes, o apreciar la presencia de un esófago de Barrett son fundamentales ${ }^{1,2}$.

El tratamiento con inhibidores de la bomba de protones logra alivio de los síntomas entre $78 \%$ a $92 \%$, pero no trata la regurgitación, no controla el reflujo no ácido y deben continuarse indefinidamente, entre el $75 \%$ y $90 \%$ de los pacientes presentan recurrencia de los síntomas o esofagitis tras su inte-
'Departamento de Cirugía Hospital Clínico Universidad de Chile. Santiago, Chile

Recibido 2021-01-25, aceptado 2021-04-14

Correspondencia a: Dr. Ítalo Braghetto M. ibraghet@hcuch.cl 
rrupción. Aún más, en pacientes refluidores asintomáticos o con síntomas atípicos, también deben ser incluidos en un plan terapéutico. Los efectos adversos de la cirugía asociado a los efectos secundarios del uso crónico de IBP motivan las terapias endoscópicas. En la literatura se mencionan una serie de efectos colaterales tales como: reducción del efecto antitrombótico del clopidogrel, incremento del riesgo de osteoporosis y fracturas, incremento del riesgo de neumonía adquirida en la comunidad y neumonía nosocomial, incremento del riesgo de infecciones por $C$. diffícile y otras infecciones entéricas, eventos perinatales y posnatales adversos con el uso durante el embarazo, nefritis intersticial aguda, deficiencia de vitamina B12, hipomagnesemia, inhibición de la absorción de hierro, incremento en el riesgo de neoplasias, aumento en el riesgo de peritonitis bacteriana espontánea en pacientes con cirrosis, neutropenia son algunas de las que se señalan en la literatura ${ }^{3}$. Por otro lado, la cirugía laparoscópica antirreflujo, si bien presenta mínimas complicaciones y casi nula mortalidad, representa un riesgo.

Los procedimientos endoluminales se iniciaron en los 80 con la idea de eliminar el uso continuo de inhibidores de bomba de protones (IBP) y minimizar la necesidad de cirugía antirreflujo ${ }^{4,5}$. Las opciones se resumen en:

1. Terapia por radiofrecuencia (Stretta).

2. Funduplicatura transoral endoscópica (FTE).

3. Mucosectomía endoscópica antirreflujo (MEAR).

4. Implantación o inyección submucosa.

Es muy importante establecer la manera de presentación clínica de la ERGE con el objetivo de ofrecer la terapia definitiva ${ }^{2-6}$. Según esto, podríamos diferenciar situaciones clínicas con comportamiento terapéutico médico o quirúrgico diferente estableciendo los buenos y malos candidatos para cirugía. A su vez pacientes con $\mathrm{HH}$ mayores a $3 \mathrm{~cm}$ son malos candidatos a terapia endoscópica ${ }^{7-9}$.

El objetivo del presente artículo es presentar un análisis crítico de los resultados reportados en la literatura internacional en cuanto a calidad de la información, específicamente enfocado a la eficacia a corto y largo plazo en paciente en los cuales se ha indicado este tipo de procedimientos.

\section{Materiales y Método}

Se efectuó una revisión exhaustiva de los artículos más relevantes en el tema indexados en Pubmed, Medline y Scielo en los últimos 10 años revisando un total de 55 trabajos de los cuales se eligieron aquellos que reportaban resultados de revisiones, series prospectivas y se excluyeron casos clínicos o videos de técnica operatoria ${ }^{10-40}$. No se encontraron trabajos referentes al tema en la literatura latinoamericana (Scielo). Se buscó específicamente los datos en cuanto a mejoría de los síntomas y calidad de vida (GIQLI o GERD-HRQL score), evaluación endoscópica, manometría, pHmetría de $24 \mathrm{~h}$, radiología y posibles complicaciones.

\section{Terapia por radiofrecuencia}

Los mecanismos propuestos incluyen la hipertrofia de la muscularis propia, la reducción de las relajaciones transitorias, la fibrosis del esfínter gastroesofágico (EGE), estos se consideran como los mecanismos de acción, sin embargo, no está completamente demostrado ${ }^{11-21}$.

Un estudio prospectivo reportó normalización de la calidad de vida y síntomas de reflujo en cerca del $70 \%-80 \%$, reducción del $50 \%$ del uso de IBP en el $64 \%$ de los pacientes. Sin embargo, no hay muchos en cuanto mejoría de manometría determinado por el efecto sobre la presión de reposo del esfínter esofágico inferior $\mathrm{o} \mathrm{pH}$ metría posoperatoria con reducción del porcentaje de tiempo de $\mathrm{pH}$ ácido menor de 4 o del score de DeMeester ${ }^{22}$ (Tabla 1).

La calidad de la evidencia de 4 ensayos comparando Stretta versus tratamiento con IBP o Nissen es deficiente y los resultados no mostraron diferencias significativas $^{23}$. Hay pocos estudios prospectivos comparando la fundoplicatura de Nissen con Stretta, aunque ambos son capaces de disminuir los síntomas, la cirugía laparoscópica presentó mayor eficacia en disminuir la necesidad de uso de IBP, pero el $\%$ de tiempo con $\mathrm{pH} \leq \mathrm{de} 4 \mathrm{y}$ la presión media del EGE no cambiaron. Perry ${ }^{17}$, mostró una disminución del score de DeMeester de 44,3 \pm 93 a 28,53 $\pm 33,4$ y del \% de tiempo pH menor de 4 , el que si bien disminuyó de $10,29 \pm 17,8$ a $6,51 \pm 12,5$, revela persistencia de reflujo ácido en valores patológicos. Triadafilopoulus reportó disminución de esofagitis erosiva de $53 \%$ a $17 \%$, pero la presión de reposo del EGE persistió en valores anormales (presión de reposo subió de 5,2 $\pm 0,3 \mathrm{mmHg}$ a $8,0 \pm 0,4 \mathrm{mmHg}$ $(\mathrm{p}<0,01)$ persistiendo, por lo tanto, un EGE incompetente. Con radiofrecuencia se redujo la exposición ácida de $10,6 \%$ (rango 7,8\%-13,0\%) a 6,8\% (rango $3,1 \%-9,1 \%)(\mathrm{p}<0,01)$ a $\operatorname{los} 6$ meses y a $6,3 \%$ $(4,7 \%-10,9 \%)$ a los 12 meses $(p<0,05)$. El 73\% de los pacientes se mantienen con valores límites de reflujo ácido ${ }^{18}$.

Dos recientes metaanálisis incluyendo 2.468 pacientes con seguimiento de 25,4 meses, demuestran lo mismo. Algunos sugieren que repetidas sesiones 
de radiofrecuencia podría mejorar los resultados. En definitiva, la calidad de los estudios es muy deficiente, ya que muchos estudios adolecen de evaluación manométrica, endoscópica y radiológica para sacar conclusiones válidas, sin un adecuado seguimiento a largo plazo ${ }^{2,3,19-21}$ (Tabla 1).

En resumen, los estudios proporcionan una evidencia limitada en cuanto a su eficacia. Se necesitan más estudios para determinar la eficacia de Stretta a largo plazo.

\section{Fundoplicatura transoral (FT)}

Este procedimiento que sigue los principios de la cirugía antirreflujo, es decir, al reducir una hernia de hiato $(\leq 2 \mathrm{~cm})$ y crear una válvula de 2 a $4 \mathrm{~cm}$ de longitud de al menos de $270^{\circ}$. La FT ha sufrido varias modificaciones desde su introducción y se han utilizado diferentes dispositivos para efectuar el procedimiento. (Esophy $X^{\circledR}$, Bard $^{\circledR}$ Endocinch, NDOplicator, Wilson-Cook Endoscopic Suturing Device), y recientemente se ha presentado un nuevo dispositivo plicator GERDx ${ }^{\circledR}$ ). Trad, Hunter y otros demostraron que después de FT se controlan los síntomas en un $67 \%-97 \%$, esofagitis en $100 \%$ y en $64 \%-90 \%$ de los pacientes reducción del uso de IBP $^{21-29}$.

En una revisión sistemática incluyendo 963 pacientes, los episodios de reflujo ácido evaluados con $\mathrm{pH}$-metría no mejoraron significativamente y el uso de IBP aumentó durante el seguimiento a largo plazo, sólo $30 \%$ de los pacientes dejaron completamente los IBP a los 6 años de seguimiento. Efectos adversos graves (perforación gastrointestinal, neumonía y hemorragia) ocurren en 2,4\%. Tampoco hay estudios con un gran número de enfermos ${ }^{32-37}$.

Hay solo un artículo con 12 pacientes que menciona que tiempo de exposición al ácido se redujo del $6,8 \%$ al $3,4 \%(\mathrm{p}=0,008)$ y la puntuación de DeMeester se redujo de 19 a $12(p=0,008)^{30}$.

Buenos resultados solo se logran en cardias grado I a II de Hill, hernia hiatal $\leq 2 \mathrm{~cm}$, y en pacientes con motilidad esofágica normal, es decir, en un número muy limitado de pacientes. Como se puede observar hay pocos datos publicados que mencionan el efecto manométrico y de la pHmetría posprocedimiento, menos aún estudios comparativos, por lo tanto, la evidencia es poco consistente ${ }^{22-30}$.

Otro método es el uso de Medigus ultrasonic surgical endostapler (MUSE), procedimiento sofisticado con instrumento especial y de alto costo. En un estudio multicéntrico, se encontró una mejora en la puntuación de síntomas en el 73\% de los pacientes, el $65 \%$ menor dosis de IBP a los 6 meses, pero luego debieron continuar el tratamiento, por lo tanto, una parcial eficacia. El estudio de monitoreo de $\mathrm{pH}$
Tabla 1. Análisis crítico de los resultados de los principales estudios reportados en la literatura con el uso de radiofrecuencia (Stretta) por vía endoscópica para la enfermedad por reflujo gastroesofágico

\begin{tabular}{|llc|}
\hline & & n de estudios \\
\hline Autores (n) & 12 & \\
Pacientes incluidos (n) & 736 & 8 \\
Seguimiento promedio & $6-24$ meses & 4 \\
\hline \% de pacientes con reducción & Mas de 24 meses & 6 \\
uso de IBP & No reportados & 2 \\
& $50 \%-70 \%$ & 4 \\
\hline \% de pacientes con satisfacción & Más de $70 \%$ & \\
posprocedimiento & $52 \%-86 \%$ & 9 \\
Persistencia de esofagitis endoscópica & No reportado & 3 \\
& $0 \%-28 \%$ & 9 \\
Manometría** & No reportado & 3 \\
\hline Persistencia reflujo*** & No cambios & 8 \\
& No reportado & 4 \\
\hline Estudio radiológico & Reflujo anormal & 12 \\
Complicaciones & No reportado & 8 \\
& No reportado & 4 \\
\hline
\end{tabular}

*Mejoría de síntomas. **Manometría presión de reposo EGE. ***Monitoreo $\mathrm{pH}$ de $24 \mathrm{~h}$ score DeMeester y \% de tiempo pH menor de 4 (Referencias 19-31).

esofágico de 24 h demostró disminución del reflujo ácido a los 6 meses, evidentemente un seguimiento a muy corto plazo sin ninguna validez como terapia definitiva. Aunque los datos emergentes con MUSE son alentadores, es un procedimiento relativamente nuevo con datos limitados, los resultados deben interpretarse con cautela. Un estudio multicéntrico, reporta similares resultados. La gran crítica es el número de pacientes estudiados, seguimiento muy corto, sin datos manométricos ni de monitoreo de $\mathrm{pH}$ de $24 \mathrm{~h}^{31-33}$. La Tabla 2 muestra un resumen de los resultados reportados en la literatura.

\section{Mucosectomía antirreflujo}

Este procedimiento se basa en el principio de formación de fibrosis retráctil como válvula antirreflujo. Inoue en su primera publicación con 10 pacientes, con seguimiento de 2 meses observó disminución del diámetro cardial, mejoría subjetiva y objetiva. En el último tiempo se han publicado otros trabajos, pero solo uno reporta evaluación objetiva respecto de la $\mathrm{pHmetría}{ }^{34-40}$.

Patil en India, reporta 62 pacientes, en el seguimiento se observó disfagia en $5(8 \%)$ pacientes que necesitaron una única sesión de dilatación endos- 
Tabla 2. Resultados reportados en la literatura con el uso de fundoplicatura transoral endoscópica para la enfermedad por reflujo gastroesofágico

\begin{tabular}{|c|c|c|c|c|c|c|}
\hline & EndoCinch & NDO & $\begin{array}{l}\text { Wilson-Cook } \\
\text { Plicator }\end{array}$ & GerdX System & Esophix & MUSE \\
\hline Estudios (n) & 15 & 2 & 2 & 1 & 1 & 1 \\
\hline Seguimiento & 1,6 meses- 6 años & 6-12 meses & 3-6 meses & 12 meses & 6-12 meses & 6-4 años \\
\hline Reducción uso de IBP & $32 \%-82 \%$ & $59 \%-74 \%$ & $83 \%$ & $90 \%$ & $56,5 \%$ & $90 \%$ \\
\hline Manometría** & NR & NR & No cambios & No cambios & NR & NR \\
\hline pHmetría anormal $* * *$ & $37 \%-89 \%$ & $71 \%-100 \%$ & $100 \%$ & No cambios & NR & $73 \%$ \\
\hline Radiología & NR & NR & NR & NR & NR & NR \\
\hline Complicaciones & $\begin{array}{l}4 \%-13 \% \text { menores } \\
7,4 \% \text { mayores }\end{array}$ & $\begin{array}{l}41 \% \text { menores } \\
0 \% \text { mayores }\end{array}$ & $10 \%-15 \%$ menores & NR & NR & NR \\
\hline
\end{tabular}

*\% de paciente que refieren satisfacción posprocedimiento y mejoría de síntomas (GIQLI). **Manometría presión de reposo EGE. ***\% de pacientes con score DeMeester \% de tiempo $\mathrm{pH}$ menor de 4 anormales. NR: no reportado.

cópica. La puntuación media (DE) de GERD-Q se redujo de $10,6(1,9)$ a $3,4(1,5)(p<0,001)$. Sin embargo, en $12(19,3 \%)$ pacientes se continuó con la dosis baja de IBP, mientras que $7(11,3 \%)$ pacientes continuaron con la dosis completa. Los IBP pudieron interrumpirse en $43(69,4 \%)$ pacientes.

Treinta y ocho pacientes $(61,3 \%)$ informaron telefónicamente una mejoría sintomática y dejaron de usar IBP a los 12 meses. Esofagitis se confirmó en $27,4 \%$ y $10 \%$ presentó disfagia que necesitó dilatación con balón. A los 2 meses, la puntuación DeMeester media (DE) se normalizó en $45(72,5 \%)$ pacientes de $76,8(18,3)$ a $14,3(6,1)(\mathrm{p}<0,001)$. Como se observa, los resultados no son óptimos (35). Yoo, estudió 33 pacientes, la manometría se mantuvo en valores normales (presión del EGE $16,3 \pm 8,9 \mathrm{mmHg}$ y el puntaje de DeMeester disminuyó de 11,6 preop. a 4,0 ( $p<0,001)$, lo cual significa que estos pacientes no eran grandes refluidores $^{37}$. Sumi estudió 109 pacientes, los cuales fueron sometidos a mucosectomía antirreflujo de los cuales solo 27 se controlaron con pHmetría posprocedimiento. El tiempo de exposición ácida mostró significativa reducción de 20,8 $\pm 24,3$ (preoperatorio) a $6,9 \pm 10,4$ (posoperatorio) $(\mathrm{p}<0,01)$ y el puntaje de DeMeester se redujo de 64,4 $\pm 75,7$ (preoperatorio) a $24,9 \pm 36,0$ (posoperatorio), por lo tanto el reflujo ácido persiste ${ }^{38}$.

Se critica que son muy pocos casos, seguimiento a corto plazo sin manometría ni radiología posoperatoria y lo más importante, que lo más probable es que haya estenosis fibrosa y no un esfinter funcional, lo que puede agravar el reflujo. Los datos actuales publicados son poco alentadores.

\section{Inyección o implantación submucosa}

Se usaron técnicas de inyección o implantación con diferentes materiales (Enteryx ${ }^{\circledR}$, GatekeeperTM, Plexiglas ${ }^{\circledR}$, Duraspher $\left.{ }^{\circledR}\right)$ cuyo objetivo es crear una barrera antirreflujo y disminución de diámetro cardial. No tienen un rol actual, muchas de estos instrumentos fueron discontinuados por sus complicaciones y fracaso a largo tiempo y han sido abandonados. La Tabla 3 muestra un resumen de lo reportado en la literatura. Como se puede observar los resultados no fueron satisfactorios ${ }^{4,5,10}$. Hace algunos años la FDA prohibió varios de estos procedimientos por presentar mayor morbilidad e incluso mortalidad.

La Tabla 4 muestra un resumen de los resultados reportados comparando las diversas alternativas de tratamiento endoscópico antirreflujo ${ }^{11-13,15,21}$.

\section{Conclusiones}

Frente a los datos revisados podemos concluir que:

1. Hasta el momento la terapia endoscópica no ha demostrado poder reemplazar por completo la terapia con IBP, reducen el reflujo ácido, pero no lo normalizan y han fallado en su adecuado 
Tabla 3. Resultados de los principales estudios reportados en la literatura con el uso de inyección/implantación endoscópica para la enfermedad por reflujo gastroesofágico

\begin{tabular}{|lccc|}
\hline & Enterix & Método & Plexiglas \\
Estudios (n) & 4 & 1 & 2 \\
Pacientes (n total) & 301 & 68 & 47 \\
Seguimiento (meses) & $16-12$ & 6 & 6 \\
Reducción uso de IBP (\%) & $65 \%-87 \%$ & $53 \%$ & $56 \%$ \\
Persistencia de síntomas & $75 \%$ & NR & $0 \%$ \\
Manometría* & $30 \%$ & $60 \%$ & $25 \%$ \\
Persistencia reflujo ácido** & $100 \%$ & Nejoría leve & $68 \%$ \\
Radiología & NR & NR & $26 \%$ menores \\
Complicaciones & $68 \%$ dolor & $15 \%$ menores & $3 \%$ mayores \\
\end{tabular}

NR: no reportado. *Manometría presión de reposo EGE bajo los valores normales. **Monitoreo $\mathrm{pH}$ de $24 \mathrm{~h}$ score DeMesster y \% de tiempo pH menor de 4.

Tabla 4. Resumen de los resultados reportados en la literatura con el uso de diferentes alternativas de terapia endoscópica para la enfermedad por reflujo gastroesofágico

\begin{tabular}{|c|c|c|c|c|}
\hline & $\begin{array}{c}\text { Radiofrecuencia } \\
\text { Stretta }\end{array}$ & $\begin{array}{c}\text { Fundoplicatura } \\
\text { Transoral }\end{array}$ & $\begin{array}{l}\text { Mucosectomía } \\
\text { Endoscópica }\end{array}$ & $\begin{array}{c}\text { Inyección } \\
\text { Implantación }\end{array}$ \\
\hline Estudios (n) & 12 & 15 & 5 & 4 \\
\hline Seguimiento & $\begin{array}{l}\text { 4,5 meses } \\
10 \text { años }\end{array}$ & $\begin{array}{l}\text { 1,5 meses } \\
6 \text { años }\end{array}$ & 3-10 años & 6-12 meses \\
\hline Reducción uso de IBP & $16 \%-80 \%$ & $32 \%-82 \%$ & $100 \%$ & $65 \%-87 \%$ \\
\hline Persistencia de esofagitis & $15 \%$ & $20 \%-100 \%$ & $27,4 \%$ & $100 \%$ \\
\hline Manometría (mmHg) & Leve mejoría & No cambios & $\begin{array}{l}\text { Leve aumento } \\
\quad(16,3 \pm 8,9)\end{array}$ & $\begin{array}{c}31 \% * \\
(8 \text { a } 13,8)\end{array}$ \\
\hline Persistencia reflujo** & $76 \%-80 \%$ & $11 \%-89 \%$ & $0 \%$ & $100 \% * *$ \\
\hline
\end{tabular}

NR: no reportados. *Manometría presión de reposo EGE $=$ pre, posprocedimiento. **Monitoreo pH $24 \mathrm{~h}: \%$ tiempo pH menor de 4 y Score de DeMeester elevados por sobre valores normales $(64,4 \pm 75,7$ preoperatorio a $24,9 \pm 36,0$ posoperatorio).

control, los datos manométricos y radiológicos son escasos y no conocemos las consecuencias a largo plazo

2. Los datos exitosos reportados a corto plazo se pueden explicar por el efecto placebo del procedimiento, pero los datos a largo plazo son muy variables, controversiales y no son suficientes faltando estudios comparativos. 
3. La calidad de la evidencia es deficiente puesto que el seguimiento es corto y no se sabe qué porcentaje de los pacientes fueron controlados objetivamente.

4. Muchos de los datos corresponden a estudios preliminares.

5. No hay menciones de estudios histológicos. Por lo tanto, la cirugía laparoscópica sigue siendo el estándar de oro para el tratamiento de la ERGE.

\section{Responsabilidades éticas}

Protección de personas y animales. Los autores declaran que para esta investigación no se han realizado experimentos en seres humanos ni en animales.

Confidencialidad de los datos. Los autores declaran que en este artículo no aparecen datos de pacientes.

Conflictos de interés: no hay.

\section{Bibliografía}

1. Gyawali PC, Kahrilas PJ, Savarino E, Zerbib F, Mion F, Smout A, et al. Modern diagnosis of GERD: the Lyon Consensus Gut 2018;67:1351-62.

2. Hirano I, Richter JE. Practice Parameters Committee of American College of Gastroenterology. ACG practice guidelines: esophageal reflux testing. Am J Gastroenterol. 2007;102:668-85.

3. Jaynes M, Kumar AB. The risks of longterm use of proton pump inhibitors: a critical review. Ther Adv Drug Saf. 2019;10:2042098618809927.

4. Mayor MA, Fernando HC. Endoluminal Approaches to Gastroesophageal Reflux Disease. Thorac Surg Clin. 2018;28:52732.

5. Triadafilopoulos G, Clarke JO, Hawn M. Precision GERD management for the $21 \mathrm{st}$ century Dis Esophagus 2017;30:1-6.

6. Frazzoni M, Manno M, De Micheli E, Savarino V. Efficacy in intraoesophageal acid suppression may decrease after 2-year continuous treatment with proton pump inhibitors. Dig Liver Dis. 2007;39:415-21.

7. Galindo G, Vassalle J, Marcus SN, Triadafilopoulos G. Multimodality evaluation of patients with gastroesophageal reflux disease symptoms who have failed empiric proton pump inhibitor therapy. Dis Esophagus 2013;265:443-50.

8. Kohn GP, Price RR, DeMeester SR, Zehetner J, Muensterer OJ, Awad Z. Guidelines for the management of hiatal hernia. Surg Endosc. 2013;27:4409-28.

9. Braghetto I, Korn O, Valladares H., Silva J. Azabache V. Indicaciones de cirugía antirreflujo: ¿Buenos y malos candidatos para la cirugía? Revisión bibliográfica y experiencia personal. Rev Med Chile 2010;138:605-11.

10. Raphael KL, Walsh P, Benias PC. Innovations in Endoscopic Therapy for Gastroesophageal Reflux Disease. Gastrointest Endosc Clin N Am. 2020;30:291-307.

11. Shibli F, Kitayama Y, Fass R. Novel Therapies for Gastroesophageal Reflux Disease: Beyond Proton Pump Inhibitors. Curr Gastroenterol Rep. 2020;22:16. doi: 10.1007/s11894-020-0753-y.

12. Kushner BS, Awad MM, Mikami DJ, Chand BB, Wai CJ, Murayama KM. Endoscopic treatments for GERD. Ann N Y Acad Sci. 2020;1482:121-9.

13. Patti MG. An Evidence-Based Approach to the Treatment of Gastroesophageal Reflux Disease. JAMA Surg. 2016;151:73-8.

14. Mattar SG, Qureshi F, Taylor D, Schauer PR. Treatment of refractory gastroesophageal reflux disease with radiofrequency energy (Stretta) in patients after Roux-en-Y gastric bypass. Surg Endosc.2006;20:850-4.

15. Nabi Z, Reddy DN. Endoscopic Management of Gastroesophageal Reflux Disease: Revisited. Clin Endosc. 2016;49:408-16.

16. Lipka S, Kumar A, Richter JE. No evidence for efficacy of radiofrequency ablation for treatment of gastroesophageal reflux disease: a systematic review and meta-analysis. Clin Gastroenterol Hepatol. 2015;13:1058-67.

17. Perry KA, Banerjee A, Melvin WS. Radiofrequency energy delivery to the lower esophageal sphincter reduces esophageal acid exposure and improves GERD symptoms: a systematic review and meta-analysis. Surg Laparosc Endosc Percutan Tech. 2012;22:283-8.
18. Triadafilopoulos G. Stretta. A valuable endoscopic treatment modality for gastroesophageal reflux disease. World J Gastroenterol. 2014;20:7730-8.

19. Fass R, Cahn F, Scotti DJ, Gregory DA. Systematic review and meta-analysis of controlled and prospective cohort efficacy studies of endoscopic radiofrequency for treatment of gastroesophageal reflux disease Surg Endosc. 2017;31:4865-82.

20. Sowa P, Samaraweera JB. Non ablative Radiofrequency Treatment for Gastroesophageal Reflux Disease (STRETTA). Gastrointest Endosc Clin N Am. 2020;30:253-65.

21. Coronel MA, Bernardo WM, Moura DTH, Moura ETH, Ribeiro IB, Moura EGH. The efficacy of the different endoscopic treatments versus sham, pharmacologic or surgical methods for chronic gastroesophageal reflux disease: a systematic review and meta-analysis. Arq Gastroenterol. 2018;55:296-305.

22. Richter JE, Kumar A, Lipka S, Miladinovic B, Velanovich V. Efficacy of Laparoscopic Nissen Fundoplication vs Transoral Incisionless Fundoplication or Proton Pump Inhibitors in Patients With Gastroesophageal Reflux Disease: A Systematic Review and Network Meta-analysis. Gastroenterology 2018;154:1298-308.

23. Koch OO, Kaindlstorfer A, Antoniou SA, Spaun G, Pointer R, Swanstrom LL. Subjective and objective data on esophageal manometry and impedance $\mathrm{pH}$ monitoring 1 year after endoscopic full-thickness plication for the treatment of GERD by using multiple plication implants. Gastrointest Endosc. 2013;77:714.

24. Trad KS, Barnes WE, Simoni G, 
Shughoury AB, Mavrelis G, Raza M, et al. Transoral Incisionless Fundoplication Effective in Eliminating GERD Symptoms in Partial Responders to Proton Pump Inhibitor Therapy at 6 Months: The TEMPO Randomized Clinical Trial. Surg Innov. 2015;22:26-40.

25. von Renteln D, Schmidt A, Riecken B, Caca K. Evaluating outcomes of endoscopic full-thickness plication for gastroesophageal reflux disease (GERD) with impedance monitoring. Surg Endosc. 2010;24:1040-8.

26. Hunter JG, Kahrilas PJ, Bell RC. Efficacy of transoral fundoplication vs omeprazole for treatment of regurgitation in a randomized controlled trial. Gastroenterology 2015;148:324-33.

27. Huang X, Chen S, Zhao H. Efficacy of transoral incisionless fundoplication (TIF) for the treatment of GERD: a systematic review with meta-analysis. Surg Endosc. 2017;31:1032-44.

28. Testoni PA, Testoni S, Mazzoleni G, Vailati C, Passaretti S. Long-term efficacy of transoral incisionless fundoplication with Esophyx (Tif 2.0) and factors affecting outcomes in GERD patients followed for up to 6 years: a prospective single-center study. Surg Endosc. 2015;29:2770-80.

29. Jain D, Singhal S. Transoral incisionless fundoplication for refractory gastroesophageal reflux disease: where do we stand? Clin Endosc. 2016;49:147-56.

30. Testoni S, Hassan C, Mazzoleni G,
Antonelli G, Fanti L, Passaretti, S, et al. Long-term outcomes of transoral incisionless fundoplication for gastroesophageal reflux disease: systematicreview and meta-analysis. Endosc Int Open. 2021;9:E239-E246. Published online 2021 Feb 3. doi: 10.1055/a-13222209.

31. Zacherl J, Roy-Shapira A, Bonavina L, Bapaye A, Kiesslich R, Schoppmann SF, et al. Endoscopic anterior fundoplication with the Medigus Ultrasonic Surgical Endostapler (MUSE) for gastroesophageal reflux disease: 6-month results from a multi-center prospective trial. Surg Endosc. 2015;29:220-9.

32. Rieder E, Riegler M, Simi'c A, Skrobi'c OM, Bonavina L, Gurski R, et al. Alternative therapies for GERD: a way to personalized antireflux surgery Ann. NY Acad Sci. 2018;1434:360-9.

33. Kim HJ, Kwon CI, Kessler WR, Selzer DJ, McNulty G, Bapaye A, et al. Long-term follow-up results of endoscopic treatment of gastroesophageal reflux disease with the MUSE endoscopic stapling device. Surg Endosc. 2016;30:3402-8.

34. Inoue $\mathrm{H}$, Ito $\mathrm{H}$, Ikeda $\mathrm{H}$, Sato $\mathrm{Ch}$, Sato $\mathrm{H}$, Phalanusitthepha Ch, Hayee B, Eleftheriadis N, Kudo Sh. Anti-reflux mucosectomy for gastroesophageal reflux disease in the absence of hiatus hernia: a pilot study. Ann Gastroenterol. 2014; 27:346-351.

35. Patil G, Dalal A, Maydeo A. Feasibility and outcomes of anti-reflux mucosectomy for proton pump inhibitor dependent gastroesophageal reflux disease: First Indian study (with video). Dig Endosc. 2020;32:745-52.

36. Hedberg HM, Kuchta K, Ujiki MB. First Experience with Banded Anti-reflux Mucosectomy (ARMS) for GERD: Feasibility, Safety, and Technique (with Video). J Gastrointest Surg. 2019;23:1274-8.

37. Yoo IK, Ko WJ, Kim HS, Kim HK, Kim JH, Kim WH, et al. Anti-reflux mucosectomy using a cap-assisted endoscopic mucosal resection method for refractory gastroesophageal disease: a prospective feasibility study. Surg Endosc. 2020;34:1124-31.

38. Sumi K, Inoue H, Kobayashi Y, Iwaya Y, Abad MRA, Fujiyoshi Y, et al. Endoscopic treatment of proton pump inhibitorrefractory gastroesophageal reflux disease with anti-reflux mucosectomy: Experience of 109 cases. Dig Endosc. 2021;33:34754. doi: 10.1111/den.13727. Epub 2020 Aug 11

39. Monino L, Gonzalez JM, Vitton V, Barthet M. Antireflux mucosectomy band in treatment of refractory gastroesophageal reflux disease: a pilot study for safety, feasibility and symptom control..Endosc Int Open. 2020;8:E147-E154. doi: 10.1055/a-1038-4012. Epub 2020 Jan 22.

40. Kushner BS, Awad MM, Mikami DJ, Chand BB, Wai CJ, Murayama KM. Endoscopic treatments for GERD. Ann NY Acad Sci. 2020;1482:121-9. 